\title{
03
}

\section{Two methods of amplification of coherent extreme ultraviolet radiation during harmonic generation in plasmas*}

\author{
(C) R.A. Ganeev
}

The Guo China-US Photonics Laboratory, Changchun Institute of Optics, Fine Mechanics and Physics, 130033 Changchun, China

e-mail: rashid_ganeev@mail.ru

Received December 30, 2017

Different recently revealed approaches of harmonic enhancement in plasmas are reviewed. The 4-step analytical model of resonant enhancement of high-order harmonic generation is extended to the systems possessing resonant transitions of inner-shell electrons. Role of inelastic scattering is discussed by simulation of excited state's population dynamics. The enhancement of harmonics in the In plasma using different pumps is analyzed to prove the concept. We also discuss the plasma emission and harmonic generation spectra in the case of resonance enhancement of single harmonic using various laser-produced plasmas. The analysis of these spectra showed that the enhancement of harmonics depended on the oscillator strengths of the nearby ionic transitions rather than the plasma emission transitions. Finally, we review some recent experimental studies of the phase-matching of high-order harmonic generation in multi-jet plasmas.

DOI: $10.21883 /$ OS.2018.06.46087.306-17

* Полный текст статьи опубликован в английской версии журнала. 